Article

\title{
First-Principles Study of Chemical and Topological Short-Range Orders in the Mg-Si Liquid Alloys
}

\author{
Dan Liu ${ }^{1,2 * *}$, Xunming Zhu ${ }^{2}$, Jingyu Qin ${ }^{1, *}$, Aimin Wang ${ }^{2}$, Junpeng Duan ${ }^{2}$ and Tingkun Gu ${ }^{3}$ \\ 1 Key Laboratory for Liquid-Solid Structural Evolution and Processing of Materials (Ministry of Education), \\ Shandong University, Jinan 250061, China \\ 2 Weihai Wanfeng Magnesium Industry Science and Technology Develop Co. Ltd., Weihai 264209, China; \\ zhuxm@wfjt.com (X.Z.); wangam@wfjt.com (A.W.); djp-518@163.com (J.D.) \\ 3 School of Electrical Engineering, Shandong University, Jinan 250061, China; gutk@sdu.edu.cn \\ * Correspondence: liudan2004sdu@163.com (D.L.); qinjy@sdu.edu.cn (J.Q.); \\ Tel.: +86-0631-562-5526 (D.L.); +86-0531-8839-2315 (J.Q.)
}

Academic Editor: Hugo F. Lopez

Received: 21 January 2016; Accepted: 23 March 2016; Published: 30 March 2016

\begin{abstract}
The structures of a series of Mg-Si liquid alloys were investigated by means of ab initio molecular dynamic simulation. The pair distribution function analysis manifests a tendency of aggregating for the $\mathrm{Si}-\mathrm{Si}$ pairs in the $\mathrm{Mg}_{90} \mathrm{Si}_{10}$ liquid alloy. Chemical short-range orders are observed around $\mathrm{Si}$ atoms between unlike atoms, and the maximum is observed for the eutectic $\mathrm{Mg}_{47} \mathrm{Si}_{53}$ alloy. Furthermore, the topological environment changed abruptly near the eutectic $\mathrm{Mg}_{47} \mathrm{Si}_{53}$ alloy according to Voronoi polyhedra analysis. The variation of diffusion coefficients of $\mathrm{Mg}$ and $\mathrm{Si}$ suggests that the dynamical properties of $\mathrm{Mg}-\mathrm{Si}$ liquid alloys are more sensitive to temperature than to compositions.
\end{abstract}

Keywords: Mg alloys; ab initio molecular dynamic simulation; short-range order; Voronoi polyhedron

\section{Introduction}

$\mathrm{Mg}$ alloys have exhibited great potential in the automotive and aerospace industries owing to their unique mechanical properties such as low density, high hardness, high specific strength, good vibration performance, etc. Therein, the $\mathrm{Mg}-\mathrm{Si}$ based alloys have attracted more and more attention since the 1970s when the AS41 and AS21 (Mg-Al-Si) alloys were developed [1,2]. The Mg-Si particles mainly exist in $\mathrm{Mg}-\mathrm{Al}(\mathrm{Zn})-\mathrm{Si}$ alloys in the form of $\mathrm{Mg}_{2} \mathrm{Si}$ for reinforcement. On the other hand, the high melting point of $\mathrm{Mg}_{2} \mathrm{Si}\left(1085^{\circ} \mathrm{C}\right)$ also improves the performance of $\mathrm{Mg}$ alloys at high temperature. Apart from that, as the content of $\mathrm{Si}$ increases, an $\mathrm{Mg}-\mathrm{Mg}_{2} \mathrm{Si}$ eutectic alloy forms at the composition of 1.16 atom \% Si, which improves the die-cast performance of $\mathrm{Mg}$ alloys. The strengthening mechanisms of $\mathrm{Mg}-\mathrm{Si}$ alloys had been investigated in rapidly solidified $\mathrm{Mg}-11 \mathrm{Si}-4 \mathrm{Al}$ and $\mathrm{Mg}-13 \mathrm{Si}-4 \mathrm{Al}$ alloy ribbons [3]. The results showed that the high strength is mainly attributed to fine-grain strengthening mechanism as well as the strengthening mechanisms due to $\mathrm{Mg}_{2} \mathrm{Si}$ particles. However, the strength decreases rapidly at $473 \mathrm{~K}$ due to diffusion when the $\mathrm{Mg}_{2} \mathrm{Si}$ particles are smaller than $1 \mu \mathrm{m}$. The microstructures and solidification process of an ingot metallurgy hypereutectic $\mathrm{Mg}-8 \mathrm{wt} \% \mathrm{Si}$ alloy was analyzed in experiments by means of electron probe microanalysis (EPMA) [4].

$\mathrm{Up}$ to now, most of the work on $\mathrm{Mg}-\mathrm{Si}$ has been done on solid alloys. Although Yuan et al. investigated the excess Gibbs energy of the liquid Mg-Si alloys using the Kaptay equation [5], the structures of $\mathrm{Mg}-\mathrm{Si}$ alloys before solidifying and the correlation between liquid and solid phases are still open questions. In the present work, we studied the chemical and topological short-range orders in Mg-Si melts using ab initio molecular dynamic (MD) simulations. Characteristic structures have 
been observed in eutectic and other liquid alloys. In addition, the dynamical property, i.e., diffusion coefficient, is also discussed.

\section{Materials and Methods}

The simulations were carried out using the Vienna ab initio simulation package (VASP) [6]. The interactions between ions and electrons were described by the projector augmented-wave method [7], and the electronic exchange and correlation was calculated by adopting generalized gradient approximations developed by Perdew et al. [8]. The $\Gamma$ point alone was used to sample the supercell Brillouin zone. All the simulations were performed in a canonical ensemble (NVT). The temperature was controlled using a Nosé thermostat [9] with a frequency of $52 \mathrm{ps}^{-1}$. The external pressure was adjusted by optimizing the atomic number density during the simulations to keep it within \pm 5 Kbar [10]. In addition, the cutoff energy was increased by $20 \%$ from the default value given by the VASP code to save computing time, in which case the discrepancy is negligible [11].

A cubic supercell containing 100 atoms with periodic boundary was used in simulations. The target compositions and number densities are listed in Table 1. The initial configuration is created by randomly stacking $\mathrm{Mg}$ and $\mathrm{Si}$ atoms following the defined concentration. The atomic distances of the initial configuration were first calculated, and all atom pairs with unrealistic distances were then moved apart by a given distance. The procedure was repeated until all of the interatomic distances were realistic in the configuration. The permissible distances for $\mathrm{Mg}-\mathrm{Mg}$ and $\mathrm{Si}-\mathrm{Si}$ pairs are defined as the minimum $r$ values with which the $\mathrm{g}(r)$ functions (see the definition in Section 3.1) of pure $\mathrm{Mg}$ and $\mathrm{Si}$ are zero, and for the Mg-Si pair it is defined as one half of the sum of the $r$ values of pure Mg plus $\mathrm{Si}$ [12]. The $\mathrm{Mg}_{98.84} \mathrm{Si}_{1.16}$ eutectic alloy was excluded in this work because only one $\mathrm{Si}$ atom could be included in simulations, which makes the statistic analysis unreliable. All alloys were simulated at the temperature $100 \mathrm{~K}$ above the calculated liquid line [13]. One configuration was dumped every $3 \mathrm{fs,}$ and 3000 configurations were obtained for analysis within a period of 9 ps.

Table 1. Compositions, temperatures, and number densities of investigated $\mathrm{Mg}-\mathrm{Si}$ liquid alloys.

\begin{tabular}{ccc}
\hline Composition & Temperature/K & Number Density/ $\AA^{-3}$ \\
\hline $\mathrm{Mg}_{10} \mathrm{Si}_{90}$ & 1722 & 0.0452 \\
$\mathrm{Mg}_{20} \mathrm{Si}_{80}$ & 1643 & 0.0446 \\
$\mathrm{Mg}_{30} \mathrm{Si}_{70}$ & 1548 & 0.0440 \\
$\mathrm{Mg}_{40} \mathrm{Si}_{60}$ & 1431 & 0.0434 \\
$\mathrm{Mg}_{47} \mathrm{Si}_{53}$ & 1319 & 0.0429 \\
$\mathrm{Mg}_{60} \mathrm{Si}_{40}$ & 1435 & 0.0430 \\
$\mathrm{Mg}_{67} \mathrm{Si}_{33}$ & 1458 & 0.0426 \\
$\mathrm{Mg}_{80} \mathrm{Si}_{20}$ & 1383 & 0.0419 \\
$\mathrm{Mg}_{90} \mathrm{Si}_{10}$ & 1254 & 0.0413 \\
\hline
\end{tabular}

\section{Results and Discussion}

\subsection{Pair Distribution Function}

The structure of liquid alloys is usually described using pair distribution function (PDF) which has two types, namely Faber-Ziman (F-Z) [14] and Bhatia-Thorton (B-T) [15]. The definition of F-Z PDF is:

$$
g_{\alpha \beta}(r)=\frac{L^{3}}{N_{\alpha} N_{\beta}}\left\langle\left(\sum_{i=1}^{N_{\alpha}} n_{i \beta}(r)\right) / 4 \pi r^{2} \Delta r\right\rangle
$$


where $L$ is the edge length of the simulation box, $N_{\alpha}$ and $N_{\beta}$ are the numbers of $\alpha$ and $\beta$ atoms respectively, and $n_{i \beta}$ denotes the number of $\beta$ atoms around the $i$ th $\alpha$ atom in the sphere shell from $r$ to $r+\Delta r$. By taking the concentration $c_{i}$ into account, the B-T PDF can be obtained in the form of:

$$
g_{C C}(r)=c_{\alpha} c_{\beta}\left[g_{\alpha \alpha}(r)+g_{\beta \beta}(r)-2 g_{\alpha \beta}(r)\right]
$$

which gives the chemical short-range order (CSRO) of the liquid alloy. When there is a preference between like or unlike atoms, corresponding positive or negative peaks will appear at the given distance respectively [16].

The three partial PDFs and the concentration correlation function, i.e., $g_{\mathrm{cc}}(r)$, of the $\mathrm{Mg}-\mathrm{Si}$ liquid alloys for different compositions are shown in Figure 1a-d. The first peaks of $g_{\mathrm{MgMg}}(r), g_{\mathrm{MgSi}}(r)$, and $g_{\mathrm{SiSi}}(r)$ appear at $3.15,2.73$, and $2.42 \AA$, respectively. These values are very close to the sums of corresponding atomic radii (Mg $1.60 \AA$ and Si $1.18 \AA$ ) [17], suggesting that the chemical bonding between atoms is very weak. As the Mg content increases, the second peaks of all three partial PDFs move rightward gradually. This phenomenon is thought to be related to the atomic radii difference of $\mathrm{Mg}$ and $\mathrm{Si}$ atoms. The more $\mathrm{Mg}$ atoms exist in the first coordination shell, the more space they will occupy, and, as a consequence, the farther the second coordination shell will go. Moreover, it shows that the second peaks of all partial PDFs are very weak, especially in the Si-rich area where the second peaks disappear completely. This is supposed to be connected with the relatively high simulation temperature. Due to the high melting temperature of the Si-rich alloys, atoms interact more acutely with each other, which destroyed the structure ordering from the second coordination shell. An anomaly occurs to the Si-Si partial PDF of $\mathrm{Mg}_{90} \mathrm{Si}_{10}$, as shown in Figure $1 \mathrm{~b}$ in which the first peak turns much higher and weak peaks are visible up to $\sim 9.5 \AA$, implying the formation of a medium-range order in the form of $\mathrm{Si}-\mathrm{Si}$ bonds.
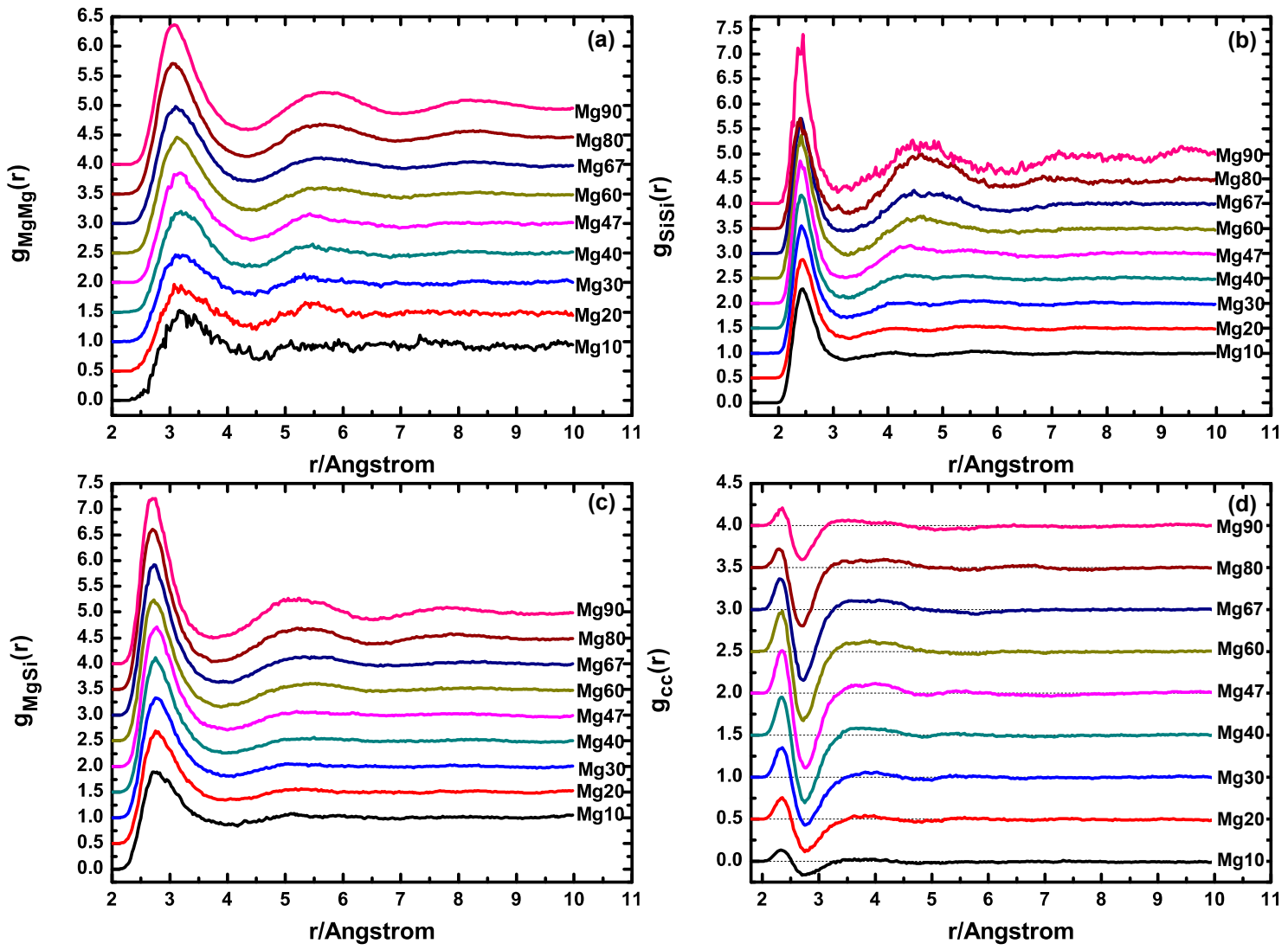

Figure 1. (a-c) Partial F-Z pair distribution functions (PDFs) and (d) B-T PDF of liquid Mg-Si alloys. 
Positive and negative peaks of the concentration correlation functions are observed at 2.32 and $2.75 \AA$, respectively, as shown in Figure 1d. According to the atomic radii, it can be easily concluded that the positive peaks correspond to $\mathrm{Si}-\mathrm{Si}$ bonds, and the negative peaks are related to $\mathrm{Mg}-\mathrm{Si}$ bonds. The Si-Si peak position here is smaller than that of $g_{\mathrm{SiSi}}(r)$ because the $g_{\mathrm{cc}}(r)$ represents the probability difference (Equation 2), and the positive peak partially superimposes with the negative peak. Apparently, the negative peaks are much stronger than the positive ones, especially than the $\mathrm{Mg}-\mathrm{Mg}$ peaks that have merged completely. In other words, compared with the $\mathrm{Mg}-\mathrm{Mg}$ and $\mathrm{Si}-\mathrm{Si}$ pairs, the Mg-Si pairs are in the majority in the liquid alloys.

A snapshot of the $\mathrm{Mg}_{90} \mathrm{Si}_{10}$ configurations is displayed in Figure 2a, where the Si atoms show a significant tendency of aggeregating. To have a deeper insight of the structure of the $\mathrm{Mg}_{90} \mathrm{Si}_{10}$ liquid alloy, we performed Fourier Transform to the 3000 configurations and built the reciprocal space. Unlike the crystallines, there is only short-range order (SRO) in liquid and amorphous alloys. The SRO manifests as a number of broad peaks that randomly distribute in the reciprocal space. There is no significant difference between different planes; therefore, an arbitrary section was drawn and shown in Figure 2b. Two typical broad peaks are indicated by arrows, along which the peaks were sliced, and the 1 dimentional profiles were plotted in the insets. The broad peaks strongly suggest the existence of SRO. A few maxima and minima of diffuse scattering were indicated in Figure $2 \mathrm{~b}$ using inverted triangles and triangles, respectively. Being different from both the SRO, which manifests as random distribution of diffuse scattering, and the long-range order, which shows a series of discrete sharp peaks in the reciprocal space, the diffuse scattering pattern of $\mathrm{Mg}_{90} \mathrm{Si}_{10}$ is more likely related to an intermediate state, i.e., a medium-range order structure. We emphasize that the initial configuration was created by randomly stacking $\mathrm{Mg}$ and $\mathrm{Si}$ atoms, which means that no crystalline structure was retained. Considering the high solidifying temperature of $\mathrm{Si}$, it is speculated that this phenomenon results from the crystallizing tendency of $\mathrm{Si}$ at such a low temperature.

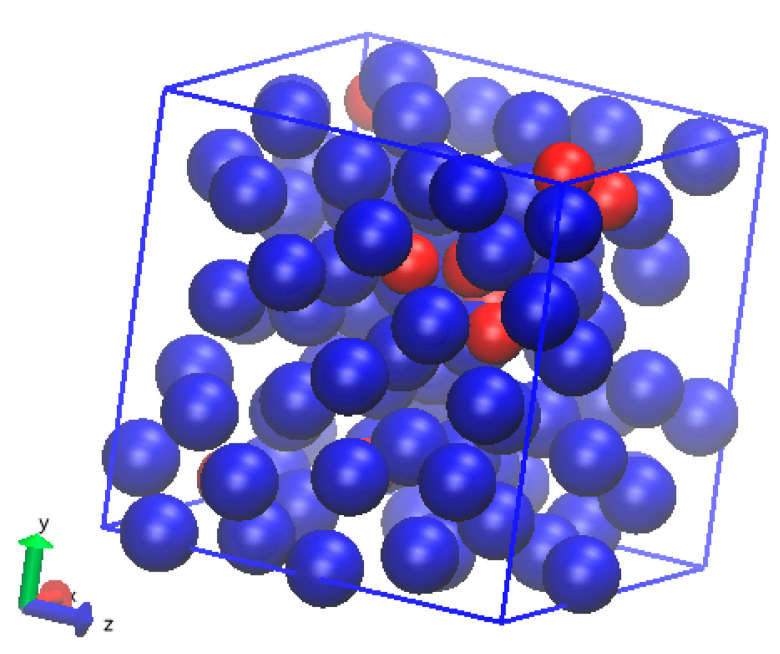

(a)

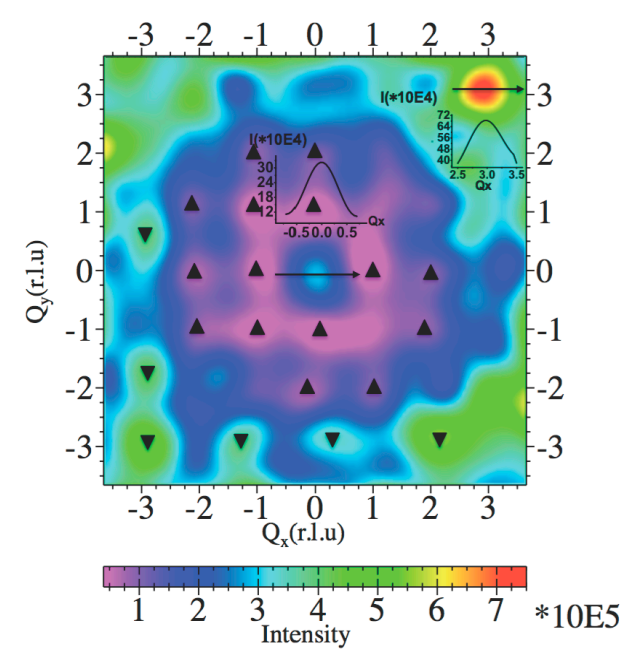

(b)

Figure 2. (a) An arbitrary snapshot of liquid $\mathrm{Mg}_{90} \mathrm{Si}_{10}$ alloy at $1254 \mathrm{~K}$; the larger balls (blue) represent $\mathrm{Mg}$ atoms, and the smaller ones (red) are Si atoms. (b) A section perpendicular to the $Q_{\mathrm{x}}-Q_{\mathrm{y}}$ plane, and $Q_{\mathrm{z}}$ equals $2 \times d_{\mathrm{Q}}$, where $d_{\mathrm{Q}}$ is the reciprocal of the box size of the reciprocal space of the liquid $\mathrm{Mg}_{90} \mathrm{Si}_{10}$ alloy at $1254 \mathrm{~K}$. The broad peaks related to the short-range order (SROs) are indicated by arrows. The inverted triangles and triangles represent the locations of maxima and minima of diffuse scattering respectively. 


\subsection{Coordination Number}

The partial coordination number $(\mathrm{CN})$ derived from the related PDF allows us to investigate the CSRO quantitatively. The average $\mathrm{CN}$ of atom $\beta$ around $\alpha$ in the first coordination shell is defined as:

$$
N_{\alpha \beta}=\int_{0}^{r_{\min }} 4 \pi r^{2} \rho_{\beta} g_{\alpha \beta}(r) \mathrm{d} r
$$

where the cutoff distance $r_{\min }$ is set at the minimum of the first peak, and $\rho_{\beta}$ is the atomic number density of $\beta$ atom. $N_{\alpha \beta}$ tells the number of $\beta$ atom around the central atom $\alpha$. The partial CNs and constitutional proportion (designated $P_{\alpha \beta}$ ) of each element are given in Table 2 . The constitutional proportion is defined by the CNs around each species: $P_{\alpha \beta}=N_{\alpha \beta} /\left(N_{\alpha \alpha}+N_{\beta \beta}\right)$.

Table 2. Partial coordination numbers (CNs) and constitutional proportions of Mg-Si liquid alloys *.

\begin{tabular}{ccccccccc}
\hline Atom \% Mg & $\boldsymbol{N}_{\mathbf{M g M g}}$ & $\boldsymbol{P}_{\mathbf{M g M g}}$ & $\boldsymbol{N}_{\mathbf{M g S i}}$ & $\boldsymbol{P}_{\mathbf{M g S i}}$ & $\boldsymbol{N}_{\mathbf{S i M g}}$ & $\boldsymbol{P}_{\mathbf{S i M g}}$ & $\boldsymbol{N}_{\mathbf{S i S i}}$ & $\boldsymbol{P}_{\mathbf{S i S i}}$ \\
\hline 10 & 1.2 & 8.9 & 12.8 & 91.1 & 0.7 & 10.0 & 6.1 & 90.0 \\
20 & 2.5 & 17.1 & 12.1 & 83.0 & 1.6 & 21.9 & 5.6 & 78.1 \\
30 & 3.4 & 26.1 & 9.6 & 73.9 & 3.1 & 34.6 & 5.8 & 65.4 \\
40 & 4.8 & 37.2 & 8.2 & 62.9 & 3.9 & 47.7 & 4.3 & 52.4 \\
47 & 6.3 & 45.4 & 7.6 & 54.6 & 4.5 & 56.4 & 3.5 & 43.6 \\
60 & 8.0 & 60.0 & 5.3 & 40.0 & 6.0 & 67.7 & 2.9 & 32.3 \\
67 & 8.4 & 67.0 & 4.1 & 33.0 & 6.8 & 75.6 & 2.2 & 24.4 \\
80 & 10.5 & 80.5 & 2.5 & 19.5 & 8.0 & 88.6 & 1.0 & 11.4 \\
90 & 11.4 & 90.7 & 1.2 & 9.4 & 8.5 & 93.6 & 0.6 & 6.5 \\
\hline
\end{tabular}

* The minor deviation in the table comes from the rounding errors.

When the $\mathrm{Mg}$ content is smaller than 47 atom \%, i.e., the eutectic composition [13], the $P_{\mathrm{MgMg}}$ is always smaller than the nominal proportion; however, when it is higher than 47 atom \%, the $P_{\mathrm{MgMg}}$ is almost equal to or slightly higher than the nominal proportion. Compared with $\mathrm{Mg}$ atoms, the $P_{\mathrm{SiMg}}$ shows larger discrepancy from the nominal proportion, and the largest deviation appears at 47 atom $\%$ $\mathrm{Mg}$ at which composition the $P_{\mathrm{SiMg}}$ is 56.4 atom \%. The results indicate that the CSROs mainly exist around $\mathrm{Si}$ atoms in the form of $\mathrm{Si}-\mathrm{Mg}$ pairs, and the strongest CSRO arises in the eutectic alloy, which is different from the previous results of $\mathrm{Mg}-\mathrm{Cu}$ [10] but consistent with those of the $\mathrm{Mg}-\mathrm{Zn}$ liquid alloys [18]. Furthermore, the variation of CNs also suggests a change of topological environments around both the $\mathrm{Mg}$ and $\mathrm{Si}$ atoms.

\subsection{Voronoi Polyhedron}

Voronoi polyhedron analysis is carried out using a self-developed program in which the periodic boundary condition was considered based on the algorithm proposed by Medvedev [19]. Figure 3a-c illustrate the fractions of the ten major Mg-centered Voronoi polyhedra with respect to Mg compositions. Figure $3 \mathrm{~d}$ shows the $\mathrm{CN}$ distribution of $\mathrm{Mg}$ derived from Voronoi polyhedron analysis. In general, the Voronoi polyhedra can be classified into three groups according to their trends with respect to Mg compositions. Figure 3a shows the polyhedra that decrease abruptly at the eutectic composition. On the contrary, Figure $3 \mathrm{~b}$ displays the variation of $<0,4,4,2>$ polyhedron that increases drastically at the eutectic position. The other polyhedra with small change are shown in Figure 3c. Similar results are observed for the ten major Si-centered polyhedra as displayed in Figure 4a-c except that the polyhedron shown in Figure $4 \mathrm{c}$ increases almost linearly as a function of $\mathrm{Mg}$ compositions and decreases at the composition of 90 atom \% Mg. 

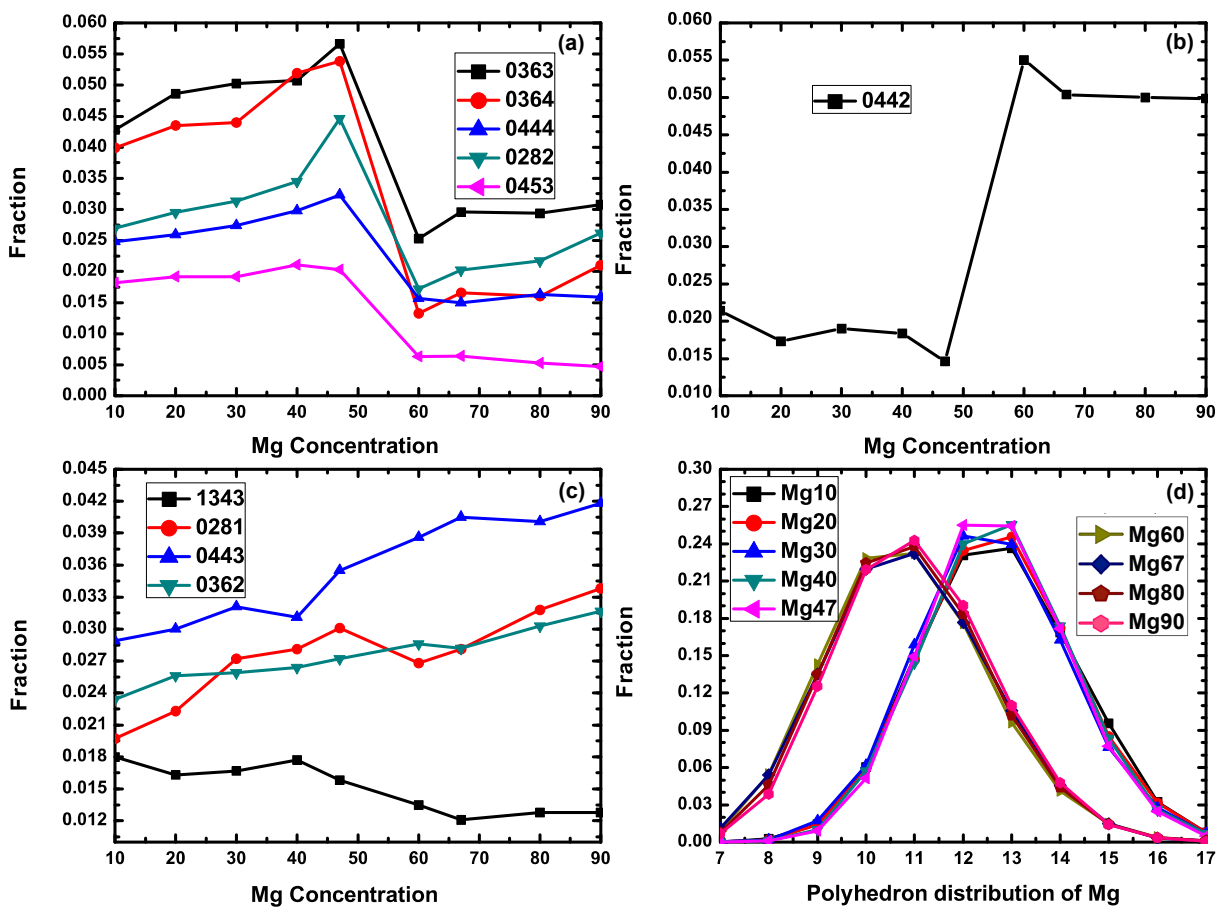

Figure 3. (a)-(c) Variation of Mg-centered Voronoi polyhedra with respect to $\mathrm{Mg}$ content and (d) coordination number $(\mathrm{CN})$ distribution of $\mathrm{Mg}$ derived from Voronoi polyhedron.
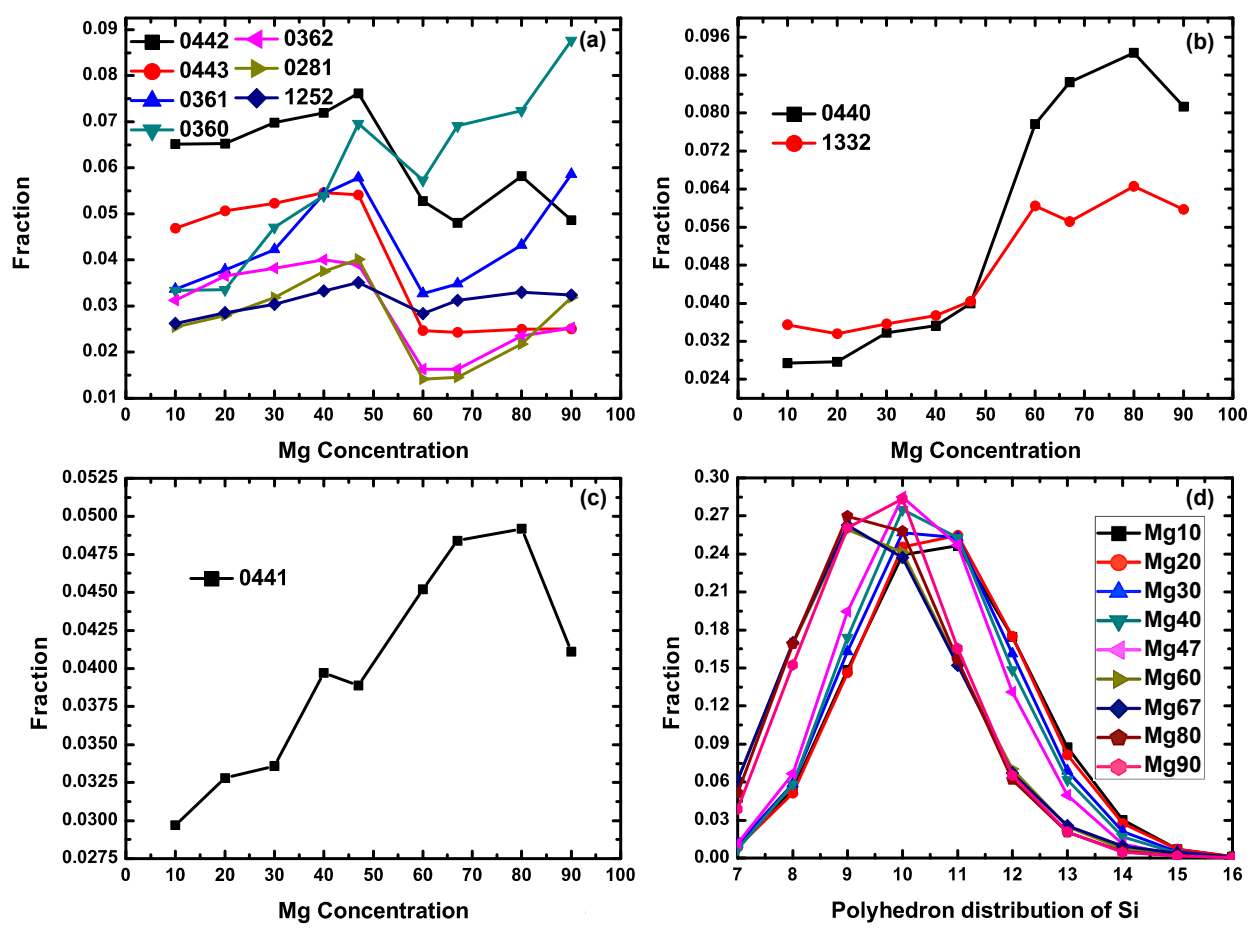

Figure 4. (a-c) Variation of Si-centered Voronoi polyhedra with respect to Mg content and (d) $\mathrm{CN}$ distribution of Si derived from Voronoi polyhedron.

Further analysis manifests that the CNs of decreasing polyhedra are mainly 12 and 13 , the polyhedra with slight variation have a $\mathrm{CN}$ of 11 , and the abruptly increasing $<0,4,4,2>$ polyhedron has 10 neighboring atoms. For the Si-centered polyhedra, the CNs of increasing polyhedra are mainly 10 and 11, and the CNs of decreasing polyhedra are mainly 8 and 9 . This was validated by the $\mathrm{CN}$ distribution of $\mathrm{Mg}$ atom and $\mathrm{Si}$ atom, as shown in Figures $3 \mathrm{~d}$ and $4 \mathrm{~d}$, respectively. The major CNs of $\mathrm{Mg}$ atoms are 12, 13 in the Si-rich region ( $\mathrm{Si}$ atom \% $\geqslant 53 \%$ ) and 10, 11 in the Mg-rich region 
( $\mathrm{Mg}$ atom $\% \geqslant 60 \%$ ), respectively. In the case of $\mathrm{Si}$, the major $\mathrm{CN}$ in the Si-rich region is 9 , and in the $\mathrm{Mg}$-rich region is 10. The result demonstrates an abrupt change of topological SRO in the liquid $\mathrm{Mg}-\mathrm{Si}$ alloys at the eutectic composition. As discussed in the $\mathrm{Mg}-\mathrm{Cu}$ work, the increase of $\mathrm{Mg}$ composition reduces the $\mathrm{CNs}$ due to the radius difference $\left(R_{\mathrm{Mg}}>R_{\mathrm{Cu}}\right)$ [10]. While the $\mathrm{Mg}$ content increases, some of them take the positons of the $\mathrm{Si}$ atoms in the first coordination shell. Since an $\mathrm{Mg}$ atom is larger than a Si atom, less Mg atoms can be arranged around the center, which leads to a smaller $\mathrm{CN}$, whereas this model cannot explain such a sudden decrease of $\mathrm{CNs}$ within so narrow a compositional range. A detailed study around the eutectic composition is desirable for further investigation. In addition, the major $\mathrm{CNs}$ of $\mathrm{Si}$ atoms in the $\mathrm{Mg}_{90} \mathrm{Si}_{10}$ liquid alloy are 9 and 10, which are distinctly larger than that of other adjacent alloys. This can be interpreted by the anomaly of $g_{\mathrm{SiSi}}(r)$ of $\mathrm{Mg}_{90} \mathrm{Si}_{10}$ in which it shows a higher probability of finding $\mathrm{Si}$ atoms around $\mathrm{Si}$. As stated above, the $\mathrm{CN}$ will be larger when the central atom is surrounded by smaller atoms.

\subsection{Self-Diffusion Coefficient}

To study the dynamical property, we calculated the diffusion coefficient of each species using the time-dependent mean square displacement (MSD), which is defined as:

$$
\left\langle\Delta r_{\alpha}(t)^{2}\right\rangle=\frac{1}{N_{\alpha}}\left\langle\sum_{i=1}^{N_{\alpha}}\left|r_{\alpha i}\left(t+t_{0}\right)-r_{\alpha i}\left(t_{0}\right)\right|^{2}\right\rangle
$$

where the sum goes overall $N_{\alpha}$ atoms of species $\alpha, t_{0}$ is an arbitrary time origin, and the angular bracket represents a thermal average or equivalently an average over time origins. For liquid alloys, the MSD has a linear correlation with $t$ in a period of time; therefore, the diffusion coefficient $D_{\alpha}$ of species $\alpha$ can be calculated by the slope through the following equation:

$$
\left\langle\Delta r_{\alpha}(t)^{2}\right\rangle \rightarrow 6 D_{\alpha} t+B_{\alpha}
$$

where $B_{\alpha}$ is constant.

Figure 5 shows the diffusion coefficients of $\mathrm{Mg}$ and $\mathrm{Si}$ atoms with respect to $\mathrm{Mg}$ compositions. It shows that the diffusion coefficients of the two species both decrease as the $\mathrm{Mg}$ content increases. They first reach a minimum at the eutectic composition and then go up to a local maximum at $\mathrm{Mg}_{2} \mathrm{Si}$ composition. Since Mg is the larger species in the binary liquid alloys, it is easy to conclude that the increase of $\mathrm{Mg}$ content will slow the diffusion of atoms. On the other hand, the variation of diffusion coefficients is nearly consistent with the temperature we applied in the simulation as indicated in Figure 5. This result implies that the diffusion of atoms in Mg-Si liquid alloys is more sensitive to the temperature than to compositions.

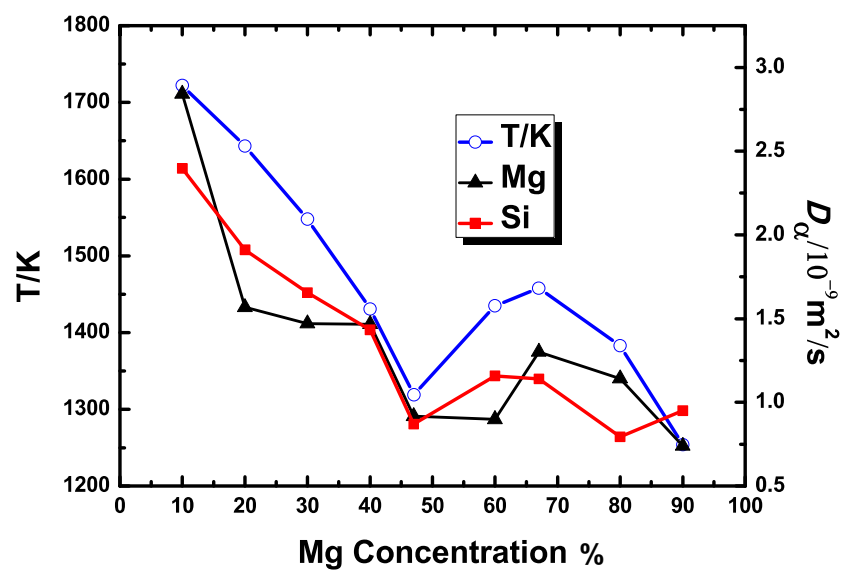

Figure 5. Diffusion coefficients of $\mathrm{Mg}$ and $\mathrm{Si}$ in the liquid $\mathrm{Mg}-\mathrm{Si}$ alloys with respect to $\mathrm{Mg}$ content. The open circles indicate the temperature applied in simulation for each alloy. 


\section{Conclusions}

The structures of liquid $\mathrm{Mg}-\mathrm{Si}$ alloys were investigated by means of ab initio MD simulation. The results of partial PDFs suggest that the chemical bonding in the liquid $\mathrm{Mg}-\mathrm{Si}$ alloys is very weak. The anomaly of the $g_{\mathrm{SiSi}}(r)$ of the $\mathrm{Mg}_{90} \mathrm{Si}_{10}$ liquid alloy is most likely due to the crystallizing tendency of $\mathrm{Si}$ atoms at low temperature. The calculated diffuse scattering in the $\mathrm{Mg}_{90} \mathrm{Si}_{10}$ liquid alloy evidences a medium-range order. This result is validated by the partial CNs of Si atoms. The Si-Mg CSRO is observed for all liquid alloys, and the maximum appears in the $\mathrm{Mg}_{47} \mathrm{Si}_{53}$ eutectic melt. Furthermore, the topological environment of the liquid alloys changes abruptly when the $\mathrm{Mg}$ content is larger than 47 atom $\% \mathrm{Mg}$. The variation of the diffusion coefficients suggests that the dynamical property of the $\mathrm{Mg}-\mathrm{Si}$ liquid alloys is mainly affected by temperature.

Acknowledgments: The financial support from the National Natural Science Foundation of China (Grant No. 51571132) is acknowledged. Thanks to Fei Wang in Missouri State University for improving the English.

Author Contributions: Jingyu Qin and Dan Liu conceived and designed the simulations; Dan Liu performed the simulations; Dan Liu, Jingyu Qin and Tingkun Gu analyzed the data; Xunming Zhu, Aimin Wang and Junpeng Duan contributed analysis tools and helpful discussions; Dan Liu wrote the paper.

Conflicts of Interest: The authors declare no conflict of interest.

\section{References}

1. Aune, T.K.; Westengen, H. Property update on magnesium die casting alloys. SAE Int. J. Mater. Manuf. 1995, 104. [CrossRef]

2. Blum, W.; Li, Y.J.; Zeng, X.H.; Zhang, P.; Von Großmann, B.; Haberling, C. Creep deformation mechanisms in high-pressure die-cast magnesium-aluminum-base alloys. Metall. Mater. Trans. A 2005, 36, 1721-1728.

3. Mabuchi, M.; Higashi, K. Strengthening mechanisms of MgSi alloys. Acta Mater. 1996, 44, 4611-4618. [CrossRef]

4. Pan, Y.; Liu, X.; Yang, H. Microstructural formation in a hypereutectic Mg-Si alloy. Mater. Charact. 2005, 55, 241-247. [CrossRef]

5. Yuan, X.; Sun, W.; Du, Y.; Zhao, D.; Yang, H. Thermodynamic modeling of the Mg-Si system with the Kaptay equation for the excess Gibbs energy of the liquid phase. Calphad 2009, 33, 673-678. [CrossRef]

6. Kresse, G.; Furthmüller, J. Efficiency of ab-initio total energy calculations for metals and semiconductors using a plane-wave basis set. Comput. Mater. Sci. 1996, 6, 15-50. [CrossRef]

7. Kresse, G.; Joubert, D. From ultrasoft pseudopotentials to the projector augmented-wave method. Phys. Rev. B 1999, 59, 1758-1775. [CrossRef]

8. Perdew, J.P.; Chevary, J.A.; Vosko, S.H.; Jackson, K.A.; Pederson, M.R.; Singh, D.J.; Fiolhais, C. Atoms, molecules, solids, and surfaces: Applications of the generalized gradient approximation for exchange and correlation. Phys. Rev. B 1992, 46, 6671-6687. [CrossRef]

9. Nosé, S. A unified formulation of the constant temperature molecular dynamics methods. J. Chem. Phys. 1984, 81, 511. [CrossRef]

10. Liu, D.; Qin, J.Y.; Gu, T.K. The structure of liquid Mg-Cu binary alloys. J. Non-Cryst. Solids 2010, 356, 1587-1592. [CrossRef]

11. Qin, J.; Gu, T.; Yang, L. Structural and dynamical properties of $\mathrm{Fe}_{78} \mathrm{Si}_{9} \mathrm{~B}_{13}$ alloy during rapid quenching by first principles molecular dynamic simulation. J. Non-Cryst. Solids 2009, 355, 2333-2338. [CrossRef]

12. Waseda, Y. The Structure of Non-Crystalline Materials: Liquids and Amorphous Solids; N. Y. McGraw-Hill: New York, NY, USA, 1980; p. 54.

13. Nayeb-Hashemi, A.A.; Clark, J.B. Magnesium-silicon binary diagram. Bull. Alloy Phase Diagr. 1990, 5, 584-592. [CrossRef]

14. Faber, T.E.; Ziman, J.M. A theory of the electrical properties of liquid metals: III. The resistivity of binary alloys. Philos. Mag. 1965, 11, 153-173. [CrossRef]

15. Bhatia, A.B.; Thornton, D.E. Structural aspects of the electrical resistivity of binary alloys. Phys. Rev. B 1970, 2, 3004-3012. [CrossRef]

16. Fischer, H.E.; Barnes, A.C.; Salmon, P.S. Neutron and X-ray diffraction studies of liquids and glasses. Rep. Prog. Phys. 2006, 69, 233-299. [CrossRef] 
17. Speight, J.G.; Lange, N.A. Lange's Handbook of Chemistry, 16th ed.; Speight, J.G., Lange, N.A., Eds.; McGraw-Hill: New York, NY, USA, 2005.

18. Dan, L. Correlation between local structures and glass forming ability of $\operatorname{liquid} \mathrm{Mg}_{x} \mathrm{Zn}_{1-x}$ Alloys. J. Non-Cryst. Solids 2016, under review.

19. Medvedev, N.N. The algorithm for three-dimensional Voronoi polyhedral. J. Comput. Phys. 1986, 67, $223-229$. [CrossRef]

(c)

(c) 2016 by the authors; licensee MDPI, Basel, Switzerland. This article is an open access article distributed under the terms and conditions of the Creative Commons by Attribution (CC-BY) license (http://creativecommons.org/licenses/by/4.0/). 